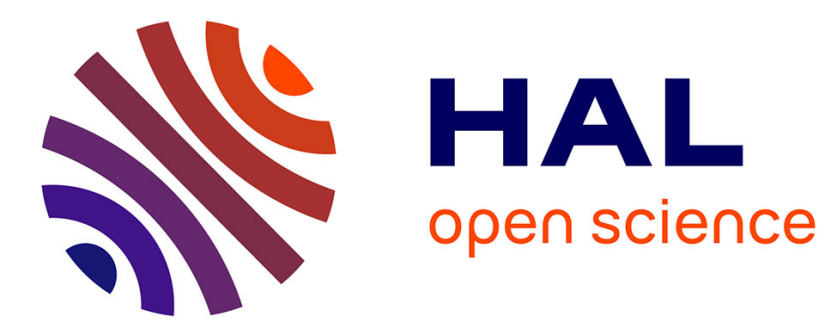

\title{
Schwarz preconditioning for high order edge element discretizations of the time-harmonic Maxwell's equations
}

Marcella Bonazzoli, Victorita Dolean, Richard Pasquetti, Francesca Rapetti

\section{To cite this version:}

Marcella Bonazzoli, Victorita Dolean, Richard Pasquetti, Francesca Rapetti. Schwarz preconditioning for high order edge element discretizations of the time-harmonic Maxwell's equations. DD23 International Conference on Domain Decomposition Methods, Jul 2015, Jeju Island, South Korea. pp.117-124, 10.1007/978-3-319-52389-7_10. hal-01250761

\section{HAL Id: hal-01250761 \\ https://hal.science/hal-01250761}

Submitted on 2 Jun 2016

HAL is a multi-disciplinary open access archive for the deposit and dissemination of scientific research documents, whether they are published or not. The documents may come from teaching and research institutions in France or abroad, or from public or private research centers.
L'archive ouverte pluridisciplinaire $\mathbf{H A L}$, est destinée au dépôt et à la diffusion de documents scientifiques de niveau recherche, publiés ou non, émanant des établissements d'enseignement et de recherche français ou étrangers, des laboratoires publics ou privés. 


\title{
Schwarz preconditioning for high order edge element discretizations of the time-harmonic Maxwell's equations
}

\author{
M. Bonazzoli ${ }^{1}$, V. Dolean ${ }^{1,2}$, R. Pasquetti ${ }^{1}$, and F. Rapetti ${ }^{1}$
}

\begin{abstract}
We focus on high order edge element approximations of waveguide problems. For the associated linear systems, we analyze the impact of two Schwarz preconditioners, the Optimized Additive Schwarz (OAS) and the Optimized Restricted Additive Schwarz (ORAS), on the convergence of the iterative solver.
\end{abstract}

\section{Introduction}

High order discretizations of PDEs for wave propagation can provide a highly accurate solution with very low dispersion and dissipation errors. The resulting linear systems can however be ill conditioned, so that preconditioning becomes mandatory. Moreover, the time-harmonic Maxwell's equations with high frequency are known to be difficult to solve by classical iterative methods, like the Helmholtz equation [3]. Domain decomposition methods are currently the most promising techniques for this class of problems (see $[1,2])$.

In order to simulate propagation in waveguide structures, we consider the second order time-harmonic Maxwell's equation:

$$
\nabla \times\left(\frac{1}{\mu} \nabla \times \mathbf{E}\right)+\left(\mathrm{i} \omega \sigma-\omega^{2} \varepsilon\right) \mathbf{E}=-\mathrm{i} \omega \mathbf{J},
$$

in the domain $\mathcal{D} \subset \mathbb{R}^{3}$ contained between two infinite parallel metallic plates $y=0$ and $y=Y$. The wave propagates in the $x$-direction and all physical

\footnotetext{
${ }^{1}$ Laboratoire J.A. Dieudonné, University of Nice Sophia Antipolis, Parc Valrose, 06108 Nice Cedex 02, France, e-mail: marcella.bonazzoli@unice.fr,victorita.dolean@unice. fr, richard.pasquetti@unice.fr, francesca.rapetti@unice.fr

2 Department of Mathematics and Statistics, University of Strathclyde, Glasgow, UK, e-mail: victorita.dolean@strath.ac.uk
} 
parameters (magnetic permeability $\mu$, electrical conductivity $\sigma$, and electric permittivity $\varepsilon$ ) are invariant in the $z$-direction. Equation (1) assumes that the electric field $\mathcal{E}(\mathbf{x}, t)=\operatorname{Re}\left(\mathbf{E}(\mathbf{x}) e^{\mathrm{i} \omega t}\right)$ has harmonic dependence on time enforced by the imposed current source $\mathcal{J}(\mathbf{x}, t)=\operatorname{Re}\left(\mathbf{J}(\mathbf{x}) e^{\mathrm{i} \omega t}\right), \omega$ being the angular frequency. We work in a bounded section $\Omega=(0, X) \times(0, Y)$ of $\mathcal{D}$ and solve the boundary value problem given by equation (1), where we set $\mathbf{J}=\mathbf{0}$, with metallic boundary conditions on the waveguide walls:

$$
\mathbf{E} \times \mathbf{n}=\mathbf{0}, \text { on } \Gamma_{\mathrm{w}}=\{y=0, y=Y\},
$$

and impedance boundary conditions at the waveguide entrance and exit:

$$
\begin{aligned}
& (\nabla \times \mathbf{E}) \times \mathbf{n}+\mathrm{i} \kappa \mathbf{n} \times(\mathbf{E} \times \mathbf{n})=\mathbf{g}^{\text {in }}, \text { on } \Gamma_{\text {in }}=\{x=0\}, \\
& (\nabla \times \mathbf{E}) \times \mathbf{n}+\mathrm{i} \kappa \mathbf{n} \times(\mathbf{E} \times \mathbf{n})=\mathbf{g}^{\text {out }}, \text { on } \Gamma_{\text {out }}=\{x=X\},
\end{aligned}
$$

$\kappa=\omega \sqrt{\varepsilon \mu}$ being the wavenumber and $\mathbf{n}=\left(n_{x}, n_{y}, 0\right)$ the outward normal to $\Gamma=\partial \Omega$. The assumptions on $\Omega$ and on the physical parameters distribution are such that $\mathbf{E}=\left(E_{x}, E_{y}, 0\right)$, which yields $\nabla \times \mathbf{E}=\left(0,0, \partial_{x} E_{y}-\partial_{y} E_{x}\right)$.

The variational formulation of the problem is: find $\mathbf{E} \in V$ such that

$$
\begin{aligned}
\int_{\Omega}[\mu \vartheta \mathbf{E} \cdot \mathbf{v}+(\nabla \times \mathbf{E}) \cdot(\nabla \times \mathbf{v})] & +\int_{\Gamma_{\text {in }} \cup \Gamma_{\text {out }}} \mathrm{i} \kappa(\mathbf{E} \times \mathbf{n}) \cdot(\mathbf{v} \times \mathbf{n}) \\
= & \int_{\Gamma_{\text {in }}} \mathbf{g}^{\text {in }} \cdot \mathbf{v}+\int_{\Gamma_{\text {out }}} \mathbf{g}^{\text {out }} \cdot \mathbf{v}, \quad \forall \mathbf{v} \in V,
\end{aligned}
$$

with $V=\left\{\mathbf{v} \in H(\operatorname{curl}, \Omega), \mathbf{v} \times \mathbf{n}=0\right.$ on $\left.\Gamma_{\mathrm{w}}\right\}$, where $H(\operatorname{curl}, \Omega)$ is the space of square integrable functions whose curl is also square integrable, $\vartheta=\mathrm{i} \omega \sigma-$ $\omega^{2} \varepsilon$, and $\mu$ is supposed constant. To write a finite element discretization of this problem we introduce a triangulation $\mathcal{T}_{h}$ of $\Omega$ and a finite dimensional subspace $V_{h} \subset H(\operatorname{curl}, \Omega)$. The simplest possible conformal discretization for the space $H(\operatorname{curl}, \Omega)$ is given by the low order Nédélec edge finite elements [6]: the local basis functions are associated with the oriented edges $E=\left\{v_{i}, v_{j}\right\}$ of a given triangle $T$ of $\mathcal{T}_{h}$ and they are given by

$$
\mathbf{w}^{E}=\lambda_{i} \nabla \lambda_{j}-\lambda_{j} \nabla \lambda_{i},
$$

where the $\lambda_{\ell}$ are the barycentric coordinates of a point w.r.t. the node $v_{\ell}$.

\section{High order edge finite elements}

We adopt here the high order extension of Nédélec elements presented in [7] and [8]. The definition of the basis functions is rather simple since it only involves the barycentric coordinates of the simplex. Given a multi-index 
$\mathbf{k}=\left(k_{1}, k_{2}, k_{3}\right)$ of weight $k=k_{1}+k_{2}+k_{3}$ (where $k_{1}, k_{2}, k_{3}$ are non negative integers), we denote by $\lambda^{\mathbf{k}}$ the product $\lambda_{1}^{k_{1}} \lambda_{2}^{k_{2}} \lambda_{3}^{k_{3}}$. The basis functions of polynomial degree $r=k+1$ over the triangle $T$ are defined as

$$
\mathbf{w}^{e}=\lambda^{\mathbf{k}} \mathbf{w}^{E}
$$

for all edges $E$ of the triangle $T$, and for all multi-indices $\mathbf{k}$ of weight $k$. Notice that these high order elements still yield a conformal discretization of $H(\operatorname{curl}, \Omega)$. Indeed, they are products between Nédélec elements, which are curl-conforming, and the continuous functions $\lambda^{\mathbf{k}}$.
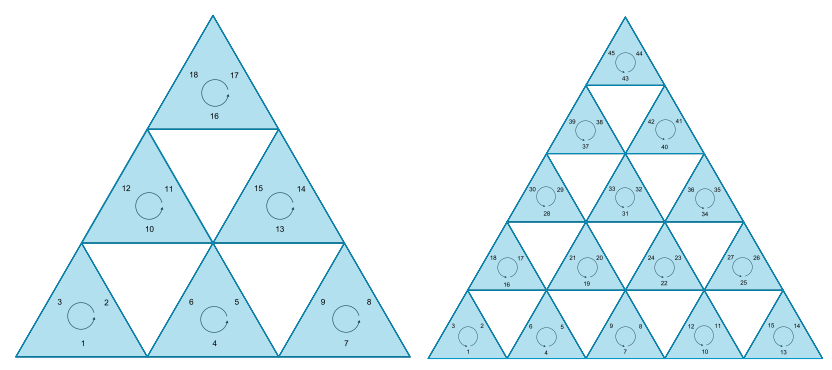

Fig. 1: The small triangles (shaded regions) and their small edges in the principal lattice of degree $r=3$ (left) and $r=5$ (right).

An interesting point of the proposed construction is the possible geometrical localization of the basis functions: the couples $\{\mathbf{k}, E\}$ appearing in (2) are in one-to-one correspondence with small edges $e$ in the principal lattice of degree $r$ of $T$ (see Fig. 1). More precisely, the small edge $e=\{\mathbf{k}, E\}$ is the small edge parallel to $E$ that belongs to the small triangle of barycentre $G$ of coordinates $\lambda_{i}(G)=\frac{1 / 3+k_{i}}{k+1}, i=1,2,3$. Thanks to the definition of the basis the circulation of each basis function along a small edge is a constant that does not depend on the triangle $T$ of the mesh.

Even if the described basis functions are very easy to generate, they don't really form a basis as they are not linearly independent. Indeed, for each small triangle which is not homothetic to the big one (the white ones in Fig. 1) one can check that the sum of the basis functions associated with its small edges is zero. Hence a redundant function should be eliminated for each 'reversed' small triangle.

\section{Schwarz preconditioning}

As shown numerically in [7], the matrix of the linear system resulting from the described high order discretization is ill conditioned. Therefore, we use and 
compare two domain decomposition preconditioners, the Optimized Additive Schwarz (OAS) and the Optimized Restricted Additive Schwarz (ORAS)

$$
M_{\mathrm{OAS}}^{-1}=\sum_{s=1}^{N_{\mathrm{sub}}} R_{s}^{T} A_{s}^{-1} R_{s}, \quad M_{\mathrm{ORAS}}^{-1}=\sum_{s=1}^{N_{\mathrm{sub}}} \widetilde{R}_{s}^{T} A_{s}^{-1} R_{s},
$$

where $N_{\text {sub }}$ is the number of overlapping subdomains $\Omega_{s}$ into which the domain $\Omega$ is decomposed. The matrices $A_{s}$ are the local matrices of the subproblems with impedance boundary conditions $(\nabla \times \mathbf{E}) \times \mathbf{n}+\mathrm{i} \kappa \mathbf{n} \times(\mathbf{E} \times \mathbf{n})$ as transmission conditions between subdomains.

In order to describe the matrices $R_{s}, \widetilde{R}_{s}$, let $\mathcal{N}$ be the set of degrees of freedom and $\mathcal{N}=\bigcup_{s=1}^{N_{\text {sub }}} \mathcal{N}_{s}$ its decomposition into the subsets corresponding to different subdomains. The matrix $R_{s}$ is a $\# \mathcal{N}_{s} \times \# \mathcal{N}$ boolean matrix, which is the restriction matrix from $\Omega$ to the subdomain $\Omega_{s}$. Its $(i, j)$ entry is equal to 1 if the $i$-th degree of freedom in $\Omega_{s}$ is the $j$-th one in the whole $\Omega$. Notice that $R_{s}^{T}$ is then the extension matrix from the subdomain $\Omega_{s}$ to $\Omega$. The matrix $\widetilde{R}_{s}$ is a $\# \mathcal{N}_{s} \times \# \mathcal{N}$ restriction matrix, like $R_{s}$, but with some of the unit entries associated with the overlap replaced by zeros: this would correspond to a decomposition into non overlapping subdomains $\widetilde{\Omega}_{s} \subset \Omega_{s}$ (completely non overlapping, not even on their border!) (see [4]). This way $\sum_{s=1}^{N_{\text {sub }}} \widetilde{R}_{s}^{T} R_{s}=I$, that is the matrices $\widetilde{R}_{s}$ give a discrete partition of unity (which is made only of 1 and 0 ).

\section{Numerical results}

We present the results obtained for a waveguide with $X=0.0502 \mathrm{~m}$, $Y=0.00254 \mathrm{~m}$, with the physical parameters: $\varepsilon=\varepsilon_{0}=8.85 \cdot 10^{-12} \mathrm{~F} \mathrm{~m}^{-1}$, $\mu=\mu_{0}=1.26 \cdot 10^{-6} \mathrm{H} \mathrm{m}^{-1}$ and $\sigma=0.15 \mathrm{~S} \mathrm{~m}^{-1}$. We consider three angular frequencies $\omega_{1}=16 \mathrm{GHz}, \omega_{2}=32 \mathrm{GHz}$, and $\omega_{3}=64 \mathrm{GHz}$, which correspond to wavenumbers $\kappa_{1}=153.43 \mathrm{~m}^{-1}, \kappa_{2}=106.86 \mathrm{~m}^{-1}, \kappa_{3}=213.72 \mathrm{~m}^{-1}$, varying the mesh size $h$ according to the relation $h^{2} \cdot \kappa^{3}=2[5]$.

We solve the linear system with GMRES (with a tolerance of $10^{-6}$ ), starting with a random initial guess, which ensures, unlike a zero initial guess, that all frequencies are present in the error. We compare the ORAS and OAS preconditioners, taking a stripwise subdomains decomposition, along the wave propagation, as shown in Fig. 2. Indeed, this is a preliminary testing of the discretization method and the preconditioner on a simple geometry which is the two-dimensional rectangular waveguide propagating only one mode; in this case, it is not necessary to consider more complicated or general decompositions.

In our tests we vary the polynomial degree $r=k+1$, the angular frequency $\omega$ and so the wavenumber $\kappa$, the number of subdomains $N_{\text {sub }}$, and finally the overlap size $\delta_{\text {ovr }}$. Here, $\delta_{\text {ovr }}=h, 2 h, 4 h$ means that we consider an overlap 


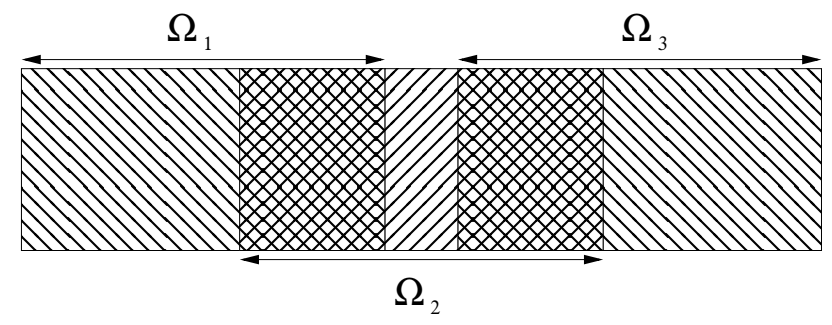

Fig. 2: The stripwise decomposition of the domain.

Table 1: Influence of $k\left(\omega=\omega_{2}, N_{\text {sub }}=2, \delta_{\text {ovr }}=2 h\right)$.

\begin{tabular}{crrcccc}
\hline$k$ & $N_{\text {dofs }}$ & $N_{\text {iterNp }}$ & $N_{\text {iter }}$ & $\max |\lambda-1|$ & $\#\{\lambda:|\lambda-1|>1\} \#\{\lambda:|\lambda-1|=1\}$ \\
\hline 0 & 282 & 179 & $5(10)$ & $1.04 e-1(1.38 e+1)$ & $0(4)$ & $0(12)$ \\
1 & 884 & 559 & $6(15)$ & $1.05 e-1(1.63 e+1)$ & $0(8)$ & $0(40)$ \\
2 & 1806 & 1138 & $6(17)$ & $1.05 e-1(1.96 e+1)$ & $0(12)$ & $0(84)$ \\
3 & 3048 & 1946 & $6(21)$ & $1.05 e-1(8.36 e+2)$ & $0(16)$ & $0(144)$ \\
4 & 4610 & 2950 & $6(26)$ & $1.05 e-1(1.57 e+3)$ & $0(20)$ & $0(220)$ \\
\hline
\end{tabular}

Table 2: Influence of $\omega\left(k=2, N_{\mathrm{sub}}=2, \delta_{\mathrm{ovr}}=2 h\right)$.

\begin{tabular}{crrcccc}
\hline$\kappa$ & $N_{\text {dofs }}$ & $N_{\text {iterNp }}$ & $N_{\text {iter }}$ & $\max |\lambda-1|$ & $\#\{\lambda:|\lambda-1|>1\} \#\{\lambda:|\lambda-1|=1\}$ \\
\hline 153.43 & 339 & 232 & $5(11)$ & $2.46 e-1(1.33 e+1)$ & $0(6)$ & $0(45)$ \\
106.86 & 1806 & 1138 & $6(17)$ & $1.05 e-1(1.96 e+1)$ & $0(12)$ & $0(84)$ \\
213.72 & 7335 & 4068 & $9(24)$ & $3.03 e-1(2.73 e+1)$ & $0(18)$ & $0(123)$ \\
\hline
\end{tabular}

Table 3: Influence of $N_{\mathrm{sub}}\left(k=2, \omega=\omega_{2}, \delta_{\mathrm{ovr}}=2 h\right)$.

\begin{tabular}{crccc}
\hline$N_{\text {sub }}$ & $N_{\text {iter }}$ & $\max |\lambda-1|$ & $\#\{\lambda:|\lambda-1|>1\} \#\{\lambda:|\lambda-1|=1\}$ \\
\hline 2 & $6(17)$ & $1.05 e-1(1.96 e+1)$ & $0(12)$ & $0(84)$ \\
4 & $10(27)$ & $5.33 e-1(1.96 e+1)$ & $0(38)$ & $0(252)$ \\
8 & $19(49)$ & $7.73 e-1(1.96 e+1)$ & $0(87)$ & $0(588)$ \\
\hline
\end{tabular}

Table 4: Influence of $\delta_{\text {ovr }}\left(k=2, \omega=\omega_{2}, N_{\text {sub }}=2\right)$.

\begin{tabular}{crccc}
\hline$\delta_{\text {ovr }}$ & $N_{\text {iter }}$ & $\max |\lambda-1|$ & $\#\{\lambda:|\lambda-1|>1\} \#\{\lambda:|\lambda-1|=1\}$ \\
\hline $1 h$ & $10(20)$ & $1.95 e+1(1.96 e+1)$ & $3(12)$ & $0(39)$ \\
$2 h$ & $6(17)$ & $1.05 e-1(1.96 e+1)$ & $0(12)$ & $0(84)$ \\
$4 h$ & $5(14)$ & $1.06 e-1(1.96 e+1)$ & $0(12)$ & $0(174)$ \\
\hline
\end{tabular}


of 1,2,4 mesh triangles along the horizontal direction. Tables $1-4$ show the total number of degrees of freedom $N_{\text {dofs }}$, the number of iterations $N_{\text {iter }}$ for convergence of GMRES preconditioned with ORAS(OAS) $\left(N_{\text {iterNp }}\right.$ refers to GMRES without any preconditioner), the greatest distance in the complex plane between $(1,0)$ and the eigenvalues of the preconditioned matrix, the number of eigenvalues that have distance greater than 1 , and the number of eigenvalues that have distance equal to 1 (up to a tolerance of $10^{-10}$ ). Indeed, if $A$ is the system matrix and $M$ is the domain decomposition preconditioner, then $I-M^{-1} A$ is the iteration matrix of the domain decomposition method used as an iterative solver. So, here we see if the eigenvalues of the preconditioned matrix $M^{-1} A$ are contained in the unitary disk centered at $(1,0)$. Notice that the matrix of the system doesn't change when $N_{\text {sub }}$ or $\delta_{\text {ovr }}$ vary, so in Tables 3-4 we don't report $N_{\text {dofs }}=1806$ and $N_{\text {iterNp }}=1138$ again. In Figs. 3 and 4 we show for certain values of the parameters the whole spectrum of the matrix preconditioned with ORAS and OAS respectively (notice that many eigenvalues are multiple).

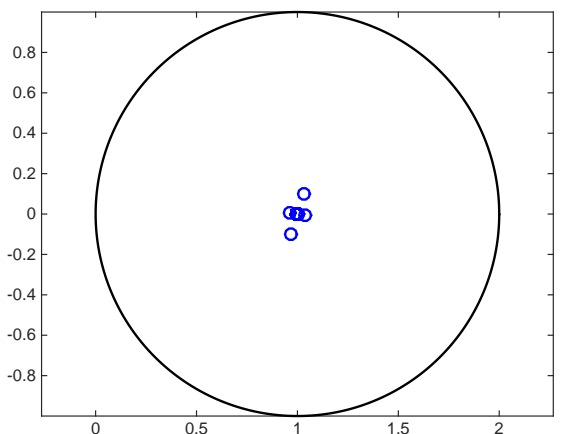

(a) $\underline{k=2, \omega_{2}, N_{\mathrm{sub}}=2, \delta_{\text {ovr }}=2 h}$

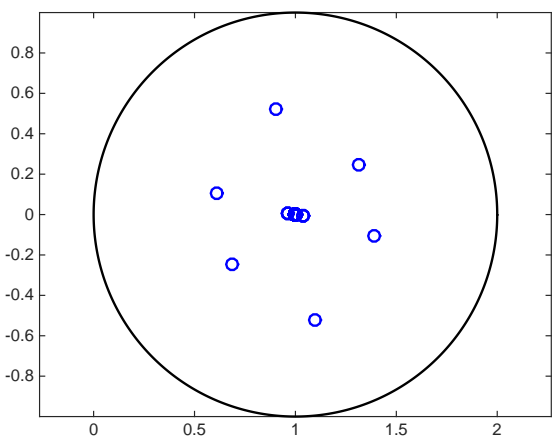

(c) $k=2, \omega_{2}, N_{\mathrm{sub}}=4, \delta_{\mathrm{ovr}}=2 h$

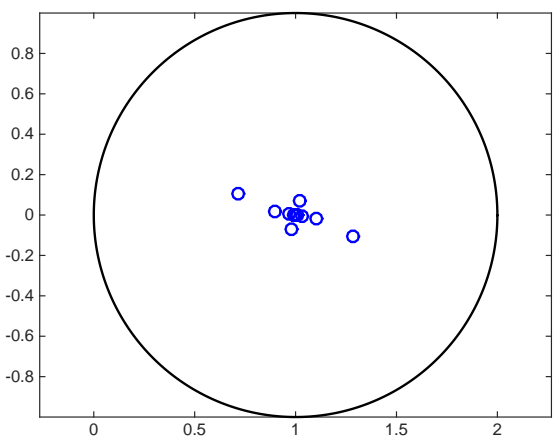

(b) $k=2, \underline{\omega_{3}}, N_{\text {sub }}=2, \delta_{\text {ovr }}=2 h$

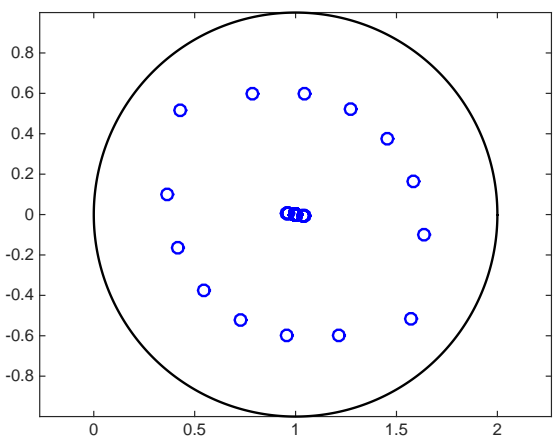

(d) $k=2, \omega_{2}, \underline{N_{\text {sub }}=8}, \delta_{\text {ovr }}=2 h$

Fig. 3: Spectrum in the complex plane of the ORAS-preconditioned matrix. 


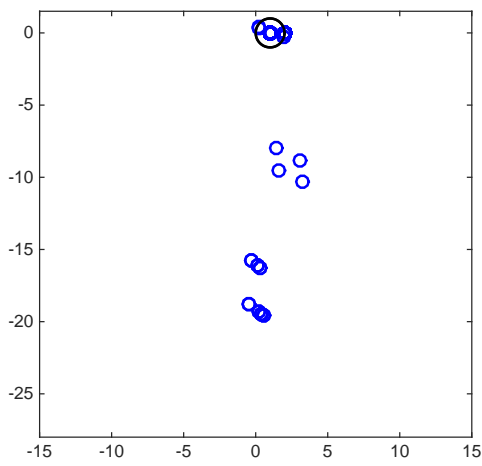

(a) $\underline{k=2, \omega_{2}, N_{\mathrm{sub}}=2, \delta_{\mathrm{ovr}}=2 h}$

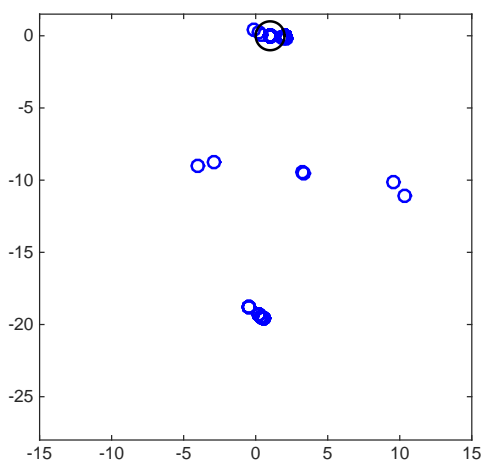

(c) $k=2, \omega_{2}, \underline{N_{\mathrm{sub}}=4,}, \delta_{\mathrm{ovr}}=2 h$

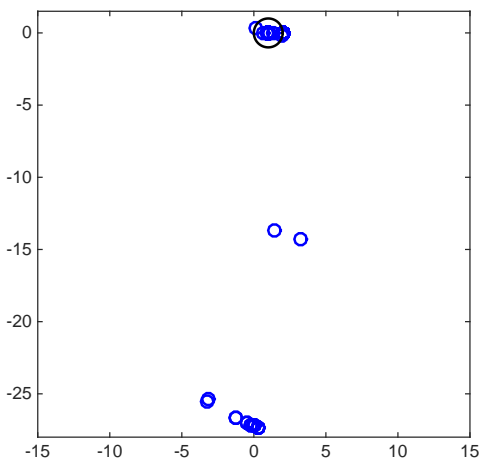

(b) $k=2, \underline{\omega_{3}}, N_{\mathrm{sub}}=2, \delta_{\mathrm{ovr}}=2 h$

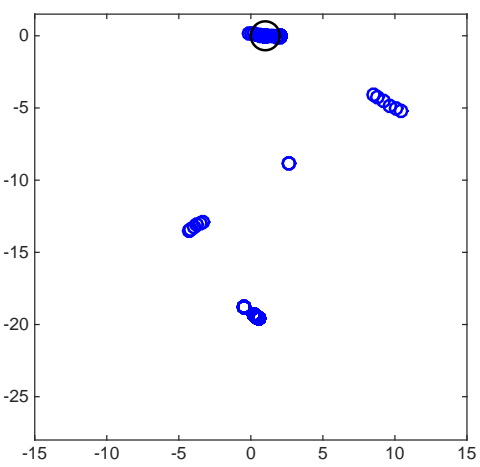

(d) $k=2, \omega_{2}, \underline{N_{\mathrm{sub}}=8}, \delta_{\mathrm{ovr}}=2 h$

Fig. 4: Spectrum in the complex plane of the OAS-preconditioned matrix.

We can see that the non preconditioned GMRES is very slow, and the ORAS preconditioning gives much faster convergence than the OAS preconditioning. Moreover, convergence becomes slower when $k, \omega$ or $N_{\text {sub }}$ increase, or when the overlap size decreases; actually, when varying $k$, the number of iterations for convergence using the ORAS preconditioner is equal to 5 for $k=0$ and then it stays equal to 6 for $k>0$.

Notice also that for 2 subdomains the spectrum is well clustered inside the unitary disk with the ORAS preconditioner, except for the case with $\delta_{\text {ovr }}=h$, in which 3 eigenvalues are outside with distances from $(1,0)$ equal to $19.5,19.4,14.4$. Then, for 4 and 8 subdomains the spectrum is not so well clustered. With the OAS preconditioner there are always eigenvalues outside the unitary disk. For all the considered cases, the less clustered the spectrum, the slower the convergence. 


\section{Conclusion}

Numerical experiments have shown that Schwarz preconditioning improves significantly the GMRES convergence for different values of physical and numerical parameters, and that the ORAS preconditioner always performs much better than the OAS preconditioner. The only advantage of the OAS method is to preserve the symmetry of the preconditioner. Finally, it has been pointed out that the spectrum of the preconditioned matrix reflects the convergence qualities, which improve when the eigenvalues are well clustered inside the unitary disk centered at $(1,0)$.

Acknowledgement This work was financed by the French National Research Agency (ANR) in the framework of the project MEDIMAX, ANR-13MONU-0012.

\section{References}

[1] V. Dolean, M. J. Gander, and L. Gerardo-Giorda. Optimized Schwarz methods for Maxwell's equations. SIAM J. Sci. Comput., 31(3):21932213, 2009.

[2] V. Dolean, M. J. Gander, S. Lanteri, J.-F. Lee, and Z. Peng. Effective transmission conditions for domain decomposition methods applied to the time-harmonic curl-curl Maxwell's equations. J. Comput. Phys., 280:232$247,2015$.

[3] O. G. Ernst and M. J. Gander. Why it is difficult to solve Helmholtz problems with classical iterative methods. In Numerical analysis of multiscale problems, volume 83 of Lect. Notes Comput. Sci. Eng., pages 325-363. Springer, Heidelberg, 2012.

[4] M. J. Gander. Schwarz methods over the course of time. Electron. Trans. Numer. Anal., 31:228-255, 2008.

[5] F. Ihlenburg and I. Babuška. Finite element solution of the Helmholtz equation with high wave number. I. The $h$-version of the FEM. Comput. Math. Appl., 30(9):9-37, 1995.

[6] J.-C. Nédélec. Mixed finite elements in $\mathbf{R}^{3}$. Numer. Math., 35(3):315-341, 1980.

[7] F. Rapetti. High order edge elements on simplicial meshes. M2AN Math. Model. Numer. Anal., 41(6):1001-1020, 2007.

[8] F. Rapetti and A. Bossavit. Whitney forms of higher degree. SIAM J. Numer. Anal., 47(3):2369-2386, 2009. 